PROCEEDINGS OF THE

AMERICAN MATHEMATICAL SOCIETY

Volume 128, Number 8, Pages 2233-2242

S 0002-9939(00)05305-3

Article electronically published on February 23, 2000

\title{
INJECTIVE RESOLUTIONS OF NOETHERIAN RINGS AND COGENERATORS
}

\author{
JUN-ICHI MIYACHI
}

(Communicated by Ken Goodearl)

\begin{abstract}
We give new construction of injective resolutions of complexes and bimodules. Applying this construction to an injective resolution of a Noetherian ring, we construct a $\Sigma$-embedding cogenerator for the category of modules of projective dimension $\leq n$. Moreover, for a Noetherian projective $k$-algebra $R$, we show that $R$ satisfies the Auslander condition if and only if the flat dimension of every $R$-module $M$ is equal to or larger than the one of the injective hull $\mathrm{E}(M)$.
\end{abstract}

\section{INTRODUCTION}

Let $R$ be a ring, and let $0 \rightarrow R \rightarrow E^{0} \rightarrow E^{1} \rightarrow \ldots$ be a minimal injective resolution of a left free module $R$ of one generator. Bass studied injective resolutions of left Noetherian rings, in particular, of commutative Noetherian rings [B1], [B2]. In the case of commutative Noetherian rings, he showed that for a prime ideal $P$ of $R$, the injective dimension of $R_{P}$ is equal to the flat dimension of the injective hull $\mathrm{E}(R / P)$ of $R / P\left[\mathrm{~B} 2\right.$, $[\mathrm{X}]$. He also showed that $R$ is Gorenstein if and only if $E^{n}$ is the direct sum of indecomposable injective $R$-modules $\mathrm{E}(R / P)$, where $P$ are prime ideals of height $n$, for every $n \geq 0$ [B2]. In other words, for every $n \geq 0, E^{n}$ is the direct sum of indecomposable injective $R$-modules $I$ of which the flat dimensions are equal to $n$. Xu studied categorical properties of Gorenstein rings $[\mathrm{X}]$. In the case of non-commutative Noetherian rings, Auslander provided the homological condition, which is called the Auslander condition, as one face of non-commutative versions of Gorenstein rings [FGR. For a non-commutative Noetherian ring $R$ satisfying the Auslander condition, Hoshino showed that every indecomposable injective left $R$-module $I$ of flat dimension $n$ is a direct summand of $E^{n}$ [H1]. By using dualities of derived categories, we showed that if the injective dimensions of ${ }_{R} R$ and $R_{R}$ are finite, then every indecomposable injective left $R$-module appears in some $E^{n}$ [Mi]. In this paper, by using a new construction of injective resolutions of complexes, we provide results concerning injective resolutions of Noetherian rings, projective dimension, flat dimension of $R$-modules. Moreover we study cogenerators for categories of modules of finite flat dimension in order to describe categorical properties of module categories.

Received by the editors June 25, 1998 and, in revised form, September 15, 1998.

1991 Mathematics Subject Classification. Primary 16D50, 16D90, 16E10, 18G35; Secondary 16D20, 18E30. 
In Section 1, from the point of view of derived categories we give a new construction of injective resolutions of complexes (Lemma 1.1). And we define $\Sigma$-embedding cogenerators for additive categories, and study the relation between an injective resolution of a module $U$ and an additive category arising from $U$ (Propositions 1.4 1.5). In Section 2, we show that $\bigoplus_{i=0}^{n} E^{i}$ is a $\Sigma$-embedding cogenerator for the category of left $R$-modules of projective dimension $\leq n$ (Theorem 2.2). As a corollary, we show that if the left injective dimension of $R$ is finite, then $\bigoplus_{i>0} E^{i}$ is a $\Sigma$-embedding cogenerator for the category of left $R$-modules of finite flat dimension (Corollary 2.3). Moreover, we get another proof of a result [Mi], Corollary 4.7 (Corollary 2.4). In Section 3, we study the case of a projective $k$-algebra $R$ over a commutative ring $k$. For a bimodule ${ }_{A} M_{B}$, we construct an injective resolution of $M$ in $\operatorname{Mod} A \otimes_{k} B^{o p}$ by using an injective resolution of $M$ in Mod $A$ (Theorem 3.5). As an application of this, we study injective resolutions of flat modules (Proposition 3.8 , and we show that $\bigoplus_{i=0}^{n} E^{i}$ is an injective cogenerator for the category of left $R$-modules of flat dimension $\leq n$ (Theorem 3.9). In Section 4, we apply results of Section 3 to projective $k$-algebras satisfying the Auslander condition. As a consequence, we get the non-commutative version of categorical properties in commutative Gorenstein rings. In particular, in the case of a Noetherian projective $k$-algebra $R$ over a commutative ring $k$, we show that $R$ satisfies the Auslander condition if and only if the flat dimension of every $R$-module $M$ is equal to or larger than the one of the injective hull $\mathrm{E}(M)$ (Theorem 4.1).

\section{INJECTIVE RESOLUTIONS OF COMPLEXES}

Throughout this paper, we assume that all rings have non-zero unity, and that all modules are unital. From the point of view of derived categories, we give the following "The piled resolution lemma". For a $\operatorname{ring} R$, we denote by $\operatorname{Mod} R$ (resp., $\bmod R, \operatorname{lnj} R$ ) the category of left (resp., finitely presented left, injective left) $R$ modules. Let $\mathcal{A}$ be an additive category, $\mathrm{C}(\mathcal{A})$ the category of complexes of objects in $\mathcal{A}, \mathrm{K}(\mathcal{A})$ the homotopy category of $\mathcal{A}$, and $\mathrm{K}^{+}(\mathcal{A}), \mathrm{K}^{-}(\mathcal{A})$ and $\mathrm{K}^{b}(\mathcal{A})$ the full subcategories of $\mathrm{K}(\mathcal{A})$ generated by bounded below complexes, bounded above complexes, bounded complexes, respectively. For an abelian category $\mathcal{A}$, let $\mathrm{D}^{*}(\mathcal{A})$ be the quotient category of $\mathrm{K}^{*}(\mathcal{A})$ by the multiplicative system of quasi-isomorphisms, where $*=$ nothing or + .

For a complex $X^{\bullet}:=\left(X^{i}, d^{i}\right)$, we define the following truncation:

$$
\tau_{\leq n} X^{\bullet}: \ldots \rightarrow X^{n-1} \rightarrow X^{n} \rightarrow 0 \rightarrow \ldots
$$

For a sequence $\left\{X_{i}^{\bullet} ; f_{i}: X_{i+1}^{\bullet} \rightarrow X_{i}^{\bullet}\right\}_{i \geq 1}$ of complexes in $\mathrm{K}(\operatorname{Mod} R)$, we have the following distinguished triangle in $\mathrm{K}(\operatorname{Mod} R)$ :

$$
X^{\bullet} \rightarrow \prod_{i} X_{i}^{\bullet} \stackrel{1 \text { shift }}{\rightarrow} \prod_{i} X_{i}^{\bullet} \rightarrow
$$

We denote $X^{\bullet}$ by $\operatorname{hlim} X_{i}^{\bullet}$, and call it the homotopy limit of the sequence. According to $[\mathrm{BN}]$, for a complex $X^{\bullet} \in \mathrm{K}(\operatorname{Mod} R), X^{\bullet}$ is isomorphic to $\operatorname{hlim} \tau_{\leq n} X^{\bullet}$ in $\mathrm{D}(\operatorname{Mod} R)$.

Lemma 1.1. Let $R$ be a ring, and $L^{\bullet}$ a complex $\ldots \rightarrow 0 \rightarrow L^{0} \rightarrow L^{1} \rightarrow L^{2} \rightarrow \ldots$ in $\mathrm{K}^{+}(\operatorname{Mod} R)$. If $0 \rightarrow L^{i} \rightarrow I_{i}^{0} \rightarrow I_{i}^{1} \rightarrow \ldots$ is an injective resolution of $L^{i}(i \geq 0)$, then there is a quasi-isomorphism from $L^{\bullet}$ to a complex of the following form in 
$\mathrm{K}^{+}(\operatorname{Mod} R):$

$$
\ldots \rightarrow 0 \rightarrow I_{0}^{0} \rightarrow \bigoplus_{i=0}^{1} I_{i}^{1-i} \rightarrow \bigoplus_{i=0}^{2} I_{i}^{2-i} \rightarrow \ldots \rightarrow \bigoplus_{i=0}^{n} I_{i}^{n-i} \rightarrow \ldots
$$

Proof. Let $I_{i}^{\bullet}$ be a complex $I_{i}^{0} \rightarrow I_{i}^{1} \rightarrow \ldots \rightarrow I_{i}^{j} \rightarrow \ldots(i \geq 0)$; then $L^{i}$ has a quasi-isomorphism to $I_{i}^{\bullet}$ in $\mathrm{D}^{+}(\operatorname{Mod} R)$. By the inductive step, we construct a complex $V_{k}^{\bullet} \in \mathrm{K}^{+}(\operatorname{Inj} R)$ which has a quasi-isomorphism $\tau_{\leq k} L^{\bullet} \rightarrow V_{k}^{\bullet}$ in $\mathrm{K}^{+}(\operatorname{Mod} R)$ as follows. First, we take $V_{0}^{\bullet}:=I_{0}^{\bullet}$. Assume we have a complex $V_{k-1}^{\bullet}$ which satisfies the above condition. Since $I_{k}^{\bullet}[-k+1]$ belongs to $\mathrm{K}^{+}(\operatorname{Inj} R)$, the quasiisomorphism $\tau_{\leq k-1} L^{\bullet} \rightarrow V_{k-1}^{\bullet}$ gives a bijection $\operatorname{Hom}_{\mathrm{K}^{+}(\operatorname{Mod} R)}\left(V_{k-1}^{\bullet}, I_{k}^{\bullet}[-k+1]\right) \rightarrow$ $\operatorname{Hom}_{\mathrm{K}^{+}(\operatorname{Mod} R)}\left(\tau_{\leq k-1} L^{\bullet}, I_{k}^{\bullet}[-k+1]\right)$. Then, for the quasi-isomorphism $L_{k} \rightarrow I_{k}^{\bullet}$ and a distinguished triangle $\tau_{\leq k} L^{\bullet} \rightarrow \tau_{\leq k-1} L^{\bullet} \rightarrow L^{k}[-k+1] \rightarrow$ in $\mathrm{K}^{+}(\operatorname{Mod} R)$, we have the following commutative diagram in $\mathrm{K}^{+}(\operatorname{Mod} R)$ :

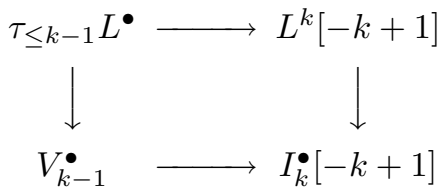

Therefore we can choose $V_{k-1}^{\bullet} \rightarrow I_{k}^{\bullet}[-k+1]$ as a map between complexes. By taking a mapping cone of $V_{k-1}^{\bullet} \rightarrow I_{k}^{\bullet}[-k+1]$, we have the following morphism between distinguished triangles in $\mathrm{K}^{+}(\operatorname{Mod} R)$ :

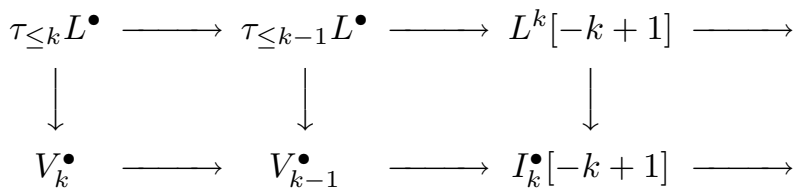

Since $V_{k}^{\bullet}$ is a mapping cone of $V_{k-1}^{\bullet} \rightarrow I_{k}^{\bullet}[-k+1]$, it is clear that $V_{k}^{\bullet}$ belongs to $\mathrm{K}^{+}(\operatorname{Inj} R)$. Since $\tau_{\leq k-1} L^{\bullet} \rightarrow V_{k-1}^{\bullet}$ and $L^{k} \rightarrow I_{k}^{\bullet}$ are quasi-isomorphisms in $\mathrm{K}^{+}(\operatorname{Mod} R), \tau_{\leq k} L^{\bullet} \rightarrow V_{k}^{\bullet}$ is a quasi-isomorphism in $\mathrm{K}^{+}(\operatorname{Mod} R)$. We have a sequence of quasi-isomorphisms from $\left\{\tau_{\leq k} L^{\bullet} ; \tau_{\leq k+1} L^{\bullet} \rightarrow \tau_{\leq k} L^{\bullet}\right\}$ to $\left\{V_{k}^{\bullet} ; V_{k+1}^{\bullet} \rightarrow\right.$ $\left.V_{k}^{\bullet}\right\}$, and then there exists a quasi-isomorphism $\alpha: \operatorname{hlim} \tau_{\leq k} L^{\bullet} \rightarrow \operatorname{hlim} V_{k}^{\bullet}$. On the other hand, by the above construction of $V_{k}^{\bullet}$, the morphism $\tau_{\leq k-1} V_{k}^{\bullet} \rightarrow \tau_{\leq k-1} V_{k-1}^{\bullet}$ is the identity in $\mathrm{C}(\operatorname{Mod} R)$ for all $k$, that is, the morphism $V_{k}^{i} \rightarrow V_{k-1}^{i}$ is the identity in $\operatorname{Mod} R$, for all $0 \leq i \leq k-1$. Therefore, there exists the inverse $\operatorname{limit} \underset{\lim }{\longleftarrow} V_{k}^{\bullet}$ which belongs to $\mathrm{K}^{+}(\operatorname{Inj} R)$. According to $[\mathrm{BN}$, Remark 2.3], there exists a quasiisomorphism $\beta: \lim _{k} V_{k}^{\bullet} \rightarrow h \lim V_{k}^{\bullet}$. Similarly, there exists a quasi-isomorphism $\gamma: L^{\bullet} \rightarrow \operatorname{hlim} \tau_{\leq k} L^{\bullet}$. Since $\varliminf_{k} V_{k}^{\bullet}$ belongs to $\mathrm{K}^{+}(\operatorname{Inj} R)$, there exists a right inverse $\tilde{\beta}$ of $\beta$ in $\mathrm{K}^{+}(\operatorname{Mod} R)$. Put $\delta:=\tilde{\beta} \alpha \gamma$; then we have the following commutative diagram in $\mathrm{K}^{+}(\operatorname{Mod} R)$ :

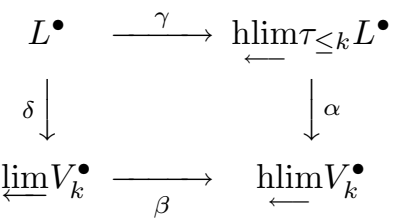




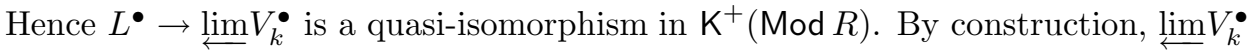
is the following form:

$$
\ldots \rightarrow 0 \rightarrow I_{0}^{0} \rightarrow \bigoplus_{i=0}^{1} I_{i}^{1-i} \rightarrow \bigoplus_{i=0}^{2} I_{i}^{2-i} \rightarrow \ldots \rightarrow \bigoplus_{i=0}^{n} I_{i}^{n-i} \rightarrow \ldots
$$

Remark 1.2. In the proof of Lemma 1.1, by direct calculation, it is not hard to see that the above morphism between distinguished triangles can be chosen to become a commutative diagram in $\mathrm{C}(\operatorname{Mod} R)$. Then we can directly construct a quasi-isomorphism $L^{\bullet} \rightarrow \underset{\lim }{\longleftarrow} V_{k}^{\bullet}$.

Corollary 1.3. Let $R$ be a ring, and $0 \rightarrow Y_{n} \rightarrow \ldots \rightarrow Y_{1} \rightarrow Y_{0} \rightarrow X \rightarrow 0$ an exact sequence of left $R$-modules. If $0 \rightarrow Y_{i} \rightarrow I_{i}^{0} \rightarrow I_{i}^{1} \rightarrow \ldots$ is an injective resolution of $Y_{i}(0 \leq i \leq n)$, then $X$ has the following injective resolution:

$$
0 \rightarrow X \rightarrow Q \rightarrow \bigoplus_{i=0}^{n} I_{i}^{i+1} \rightarrow \bigoplus_{i=0}^{n} I_{i}^{i+2} \rightarrow \ldots
$$

where $Q$ is a direct summand of $\bigoplus_{i=0}^{n} I_{i}^{i}$.

Proof. By Lemma 1.1, the complex $\ldots \rightarrow 0 \rightarrow Y_{n} \rightarrow \ldots \rightarrow Y_{1} \rightarrow Y_{0} \rightarrow 0 \rightarrow \ldots$ is isomorphic to the following complex in $\mathrm{D}^{+}(\operatorname{Mod} R)$ :

$$
V^{\bullet}: \ldots \rightarrow 0 \rightarrow V^{-n} \rightarrow V^{-n+1} \rightarrow \ldots \rightarrow V^{-1} \stackrel{d^{-1}}{\longrightarrow} V^{0} \stackrel{d^{0}}{\longrightarrow} V^{1} \rightarrow \ldots,
$$

where $V^{k}$ is isomorphic to $\bigoplus_{i=0}^{n+k} I_{i-k}^{i}$ if $0>k \geq-n$, and is isomorphic to $\bigoplus_{i=0}^{n} I_{i}^{i+k}$ if $k \geq 0$. Since $V^{k}$ is injective $(-n \leq k \leq-1)$ and $\mathrm{H}^{i} V^{\bullet}=O$ for $i \neq 0$, Im $d^{-1}$ is injective. $\mathrm{By}^{0} V^{\bullet} \cong X$, Ker $d^{0}$ is isomorphic to $X \oplus \operatorname{Im} d^{-1}$. Since the complex $V^{0} \rightarrow V^{1} \rightarrow \ldots$ is an injective resolution of $X \oplus \operatorname{Im} d^{-1}$, we get the statement.

For a left $R$-module $U$, we denote by $\operatorname{Add} U$ (resp., add $U$ ) the category of left $R$-modules which are direct summands of direct sums of copies of $U$ (resp., direct summands of finite direct sums of copies of $U$ ), and denote by $\operatorname{Res}^{n}(U)$ (resp., $\left.\operatorname{res}^{n}(U)\right)$ the full subcategory of $\operatorname{Mod} R$ (resp., $\bmod R$ ) consisting of left $R$-modules $X$ which have exact sequences $0 \rightarrow U_{n} \rightarrow \ldots \rightarrow U_{1} \rightarrow U_{0} \rightarrow X \rightarrow 0$, where $U_{i} \in \operatorname{Add} U$ (resp., add $\left.U\right)(0 \leq i \leq n)$. We denote the injective dimension of ${ }_{R} U$ (resp., the flat dimension of ${ }_{R} U$ ) by $\operatorname{idim}_{R} U$ (resp., $\operatorname{fdim}_{R} U$ ). Let $\mathcal{A}$ be an abelian category, $\mathcal{B}$ a full subcategory of $\mathcal{A}$. We call an object $X \in \mathcal{A}$ a $\Sigma$-embedding cogenerator (resp., a finitely embedding cogenerator, a cogenerator) for $\mathcal{B}$ provided that every object in $\mathcal{B}$ admits an injection to some direct sum (resp., finite direct sum, direct product) of copies of $X$ in $\mathcal{A}$.

Proposition 1.4. Let $R$ be a left coherent ring, $U$ a finitely presented left $R$ module, and $0 \rightarrow U \rightarrow E^{0} \rightarrow E^{1} \rightarrow E^{2} \rightarrow \ldots$ an injective resolution of ${ }_{R} U$. Then $\bigoplus_{i=0}^{n} E^{i}$ is a finitely embedding cogenerator for $\operatorname{res}^{n}(U)$.

Proof. Given $X \in \operatorname{res}^{n}(U)$, we have the following exact sequence:

$$
0 \rightarrow U_{n} \rightarrow \ldots \rightarrow U_{1} \rightarrow U_{0} \rightarrow X \rightarrow 0
$$

where $U_{i} \in \operatorname{add} U(n \geq i \geq 0)$. It is easy to see that every $U_{j}$ has the following injective resolution:

$$
0 \rightarrow U_{j} \rightarrow I_{j}^{0} \rightarrow I_{j}^{1} \rightarrow I_{j}^{2} \rightarrow \ldots
$$

where $I_{j}^{i} \in \operatorname{add}\left(E^{i}\right)$ for all $i \geq 0$. By Corollary 1.3, we get an injection from $X$ to $\bigoplus_{i=0}^{n} I_{i}^{i}$. Since $\bigoplus_{i=0}^{n} I_{i}^{i}$ belongs to add $\left(\bigoplus_{i=0}^{n} E^{i}\right)$, we complete the proof. 
Proposition 1.5. Let $R$ be a left Noetherian ring, $U$ a left $R$-module, and $0 \rightarrow$ $U \rightarrow E^{0} \rightarrow E^{1} \rightarrow E^{2} \rightarrow \ldots$ an injective resolution of ${ }_{R} U$. Then $\bigoplus_{i=0}^{n} E^{i}$ is a $\Sigma$-embedding cogenerator for $\operatorname{Res}^{n}(U)$.

Proof. The same as the proof of Proposition 1.4.

\section{InJeCtive RESOLUTIONS OF RINGS AND $\Sigma$-EMBEDDing COGENERATORS}

In and after this section, we fix a minimal injective resolution of a left free module $R$ of one generator:

$$
0 \rightarrow{ }_{R} R \rightarrow E^{0} \stackrel{\delta^{0}}{\longrightarrow} E^{1} \stackrel{\delta^{1}}{\longrightarrow} E^{2} \rightarrow \ldots
$$

Proposition 2.1. Let $R$ be a left coherent ring. Then $\bigoplus_{i=0}^{n} E^{i}$ is a finitely embedding cogenerator for the category of finitely presented left $R$-modules of projective dimension $\leq n$.

Proof. By Proposition 1.4

Theorem 2.2. Let $R$ be a left Noetherian ring. Then $\bigoplus_{i=0}^{n} E^{i}$ is a $\Sigma$-embedding cogenerator for the category of left $R$-modules of projective dimension $\leq n$. In particular, every injective left $R$-module of projective dimension $\leq n$ is a direct summand of $\bigoplus_{i=0}^{n} E^{i}$.

Proof. By Proposition 1.5

Corollary 2.3. Let $R$ be a left Noetherian ring. If $\operatorname{idm}\left({ }_{R} R\right)$ is finite, then $\bigoplus_{i \geq 0} E^{i}$ is a $\Sigma$-embedding cogenerator for the category of left $R$-modules of finite flat dimension.

Proof. According to [H2], Proposition 6, every left $R$-module of finite flat dimension is of finite projective dimension. Then we complete the proof by Theorem 2.2

We get another proof of a result [Mi], Corollary 4.7.

Corollary 2.4. ( $\mathrm{Mi}$, Corollary 4.7]) Let $R$ be a right coherent and left Noetherian ring. If $\operatorname{idm}\left({ }_{R} R\right)$ and $\operatorname{idm}\left(R_{R}\right)$ are at most $n$, then every indecomposable injective left $R$-module is a direct summand of $\bigoplus_{i=0}^{n} E^{i}$.

Proof. According to CE, Chap. VI, Proposition 5.6, for every finitely presented right $R$-module $M$ and every injective left $R$-module $I$, we have the following isomorphism:

$$
\begin{aligned}
\operatorname{Tor}_{i}^{R}(M, I) & \cong \operatorname{Hom}_{R}\left(\operatorname{Ext}_{R}^{i}(M, R), I\right) \\
& =0 \quad \text { for all } i>n .
\end{aligned}
$$

Therefore, the flat dimension of every indecomposable injective left $R$-module is at most $n$. By Corollary 2.3 we get the statement.

\section{InjeCtive RESOlUtions of PROJECTIVE ALgEBRAS AND COGENERATORS}

Let $k$ be a commutative ring. We call a $k$-algebra $R$ a projective $k$-algebra if $R$ is projective as a $k$-module. Let $A$ and $B$ be projective $k$-algebras. According to CE], a projective (resp., injective) $A \otimes_{k} B^{o p}$-module is projective (resp., injective) as both a right $B$-module and a left $A$-module.

Lemma 3.1. Let $R$ be a left Noetherian ring, $\left\{Q_{\lambda} ; f_{\mu, \lambda}: Q_{\lambda} \rightarrow Q_{\mu}\right\}_{\lambda \in \Lambda, \lambda \leq \mu} a$ direct system of injective left $R$-modules. Then $\underline{\lim Q_{\lambda}}$ belongs to $\operatorname{Add}\left(\bigoplus_{\lambda \in \Lambda} Q_{\lambda}\right)$. 
Proof. If $\lim Q_{\lambda}=0$, then there is nothing to prove. Otherwise, by [CE], Chap. I Ex. 8, and [Ma], $\varliminf_{\Gamma} Q_{\lambda}$ is a direct sum $\bigoplus_{\gamma \in \Gamma} I_{\gamma}$ of indecomposable injective left $R$ modules $I_{\gamma}(\gamma \in \Gamma)$. Given $\gamma \in \Gamma$, we choose a non-zero element $x$ in $I_{\gamma} \subseteq \bigoplus_{\gamma \in \Gamma} I_{\gamma}$. Let $\Lambda(x)$ be the set of pairs $(y, \lambda)$ such that $x=f_{\lambda}(y)$, where $y \in Q_{\lambda}$ for some $\lambda \in \Lambda$ and $f_{\lambda}: Q_{\lambda} \rightarrow \lim _{\lambda} Q_{\lambda}$ is the structure morphism. Since $R$ is left Noetherian and $\Lambda$ is a directed set, the set of left annihilator ideals $\operatorname{lann}(y)$ where $(y, \lambda) \in \Lambda(x)$ has a unique maximal left ideal, say, $\operatorname{lann}\left(y_{0}\right)$ where $y_{0} \in Q_{\lambda_{0}}$. Then $\operatorname{lann}(x)$ is equal to $\operatorname{lann}\left(y_{0}\right)$. By $\mathrm{Ma}, I_{\gamma}$ is a direct summand of $Q_{\lambda_{0}}$, and hence we complete the proof.

Remark 3.2. Huisgen-Zimmermann and Smalø get a similar result of Lemma 3.1 in the case of $\Sigma$-pure injective modules (see [피]).

Lemma 3.3. Let $k$ be a commutative ring, $R$ a left Noetherian projective $k$-algebra, and $0 \rightarrow R \rightarrow V^{0} \rightarrow V^{1} \rightarrow V^{2} \rightarrow \ldots$ an injective resolution of $R$ as left $R \otimes_{k} R^{o p_{-}}$ modules. If $F$ is a flat left $R$-module, then $F$ has the following injective resolution:

$$
0 \rightarrow F \rightarrow I^{0} \rightarrow I^{1} \rightarrow I^{2} \rightarrow \ldots,
$$

where $I^{i}$ belongs to $\operatorname{Add}\left({ }_{R} V^{i}\right)$ for all $i \geq 0$.

Proof. Given a flat left $R$-module $F$, we have the following acyclic complex:

$$
0 \rightarrow F \rightarrow V^{0} \otimes_{R} F \rightarrow V^{1} \otimes_{R} F \rightarrow V^{2} \otimes_{R} F \rightarrow \ldots
$$

By [L], Theorem 1.2, there is a direct system $\left\{P_{\lambda} ; f_{\mu, \lambda}: P_{\lambda} \rightarrow P_{\mu}\right\}_{\lambda \in \Lambda, \lambda \leq \mu}$ of finitely generated free left $R$-modules such that $\lim _{\lambda} P_{\lambda}$ is isomorphic to $F$. Then every $V^{i} \otimes_{R} F$ is isomorphic to $\underline{\lim } V^{i} \otimes_{R} P_{\lambda}$. By Lemma 3.1, $V^{i} \otimes_{R} F$ is an injective left $R$-module which belongs to $\operatorname{Add}\left({ }_{R} V^{i}\right)$. Hence the above acyclic complex is an injective resolution of $F$ which satisfies the statement.

Proposition 3.4. Let $k$ be a commutative ring, $R$ a left Noetherian projective $k$ algebra, and $0 \rightarrow R \rightarrow V^{0} \rightarrow V^{1} \rightarrow V^{2} \rightarrow \ldots$ an injective resolution of $R$ as left $R \otimes_{k} R^{o p}$-modules. Then $\bigoplus_{i=0}^{n} V^{i}$ is a $\Sigma$-embedding cogenerator for the category of left $R$-modules of flat dimension $\leq n$. In particular, every injective left $R$-module of flat dimension $\leq n$ is a direct summand of $\bigoplus_{i=0}^{n} R V^{i}$.

Proof. Let $X$ be a left $R$-module of flat dimension $\leq n$; then $X$ has the following flat resolution:

$$
0 \rightarrow F_{n} \rightarrow \ldots \rightarrow F_{1} \rightarrow F_{0} \rightarrow X \rightarrow 0 .
$$

By Lemma 3.3 every $F_{j}$ has the following injective resolution:

$$
0 \rightarrow F_{j} \rightarrow I_{j}^{0} \rightarrow I_{j}^{1} \rightarrow I_{j}^{2} \rightarrow \ldots
$$

where $I_{j}^{i} \in \operatorname{Add}\left(V^{i}\right)$ for all $i \geq 0$. By Corollary 1.3 we get an injection from $X$ to $\bigoplus_{i=0}^{n} I_{i}^{i}$. Since $\bigoplus_{i=0}^{n} I_{i}^{i}$ belongs to $\operatorname{Add}\left(\bigoplus_{i=0}^{n} V^{i}\right)$, we complete the proof.

Let $k$ be a commutative ring, $A$ a projective $k$-algebra. We denote by $S_{\bullet}(A)$ the bar resolution of $A$, i.e. the complex $\left(\mathrm{S}_{n}(A), d_{n+1}: \mathrm{S}_{n+1}(A) \rightarrow \mathrm{S}_{n}(A)\right)_{n \geq 0}$ such that $\mathrm{S}_{n}(A)$ is the $(n+2)$-fold tensor product over $k$ of $A$ with itself, and that $d_{n}\left(a_{0} \otimes \ldots \otimes a_{n+1}\right)=\sum_{0 \leq i \leq n+1}(-1)^{i} a_{0} \otimes \ldots \otimes a_{i} a_{i+1} \otimes \ldots \otimes a_{n+1}$. In this case, $\mathrm{S} .(A)$ is a projective resolution of $A$ in $\operatorname{Mod} A \otimes_{k} A^{o p}$. 
Theorem 3.5. Let $k$ be a commutative ring, $A$ a $k$-algebra and $B$ a projective $k$ algebra. For an $(A-B)$-bimodule $M$, let $0 \rightarrow M \rightarrow I^{0} \rightarrow I^{1} \rightarrow I^{2} \rightarrow \ldots$ be an injective resolution of $M$ as left $A$-modules. Then $M$ has the following injective resolution in $\operatorname{Mod} A \otimes_{k} B^{o p}$ :

$$
0 \rightarrow M \rightarrow V^{0} \rightarrow V^{1} \rightarrow V^{2} \rightarrow \ldots
$$

such that $V^{n}=\bigoplus_{i=0}^{n} \operatorname{Hom}_{k}\left({ }_{B} \mathrm{~S}_{i-1}(B)_{k},{ }_{A} I_{k}^{n-i}\right)$ for all $n \geq 0$, where $\mathrm{S}_{-1}(B)=B$.

Proof. It is easy to see that we have the following exact sequence in $\operatorname{Mod} A \otimes_{k} B^{o p}$ :

$$
0 \rightarrow \operatorname{Hom}_{B}\left({ }_{B} B_{B},{ }_{A} M_{B}\right) \rightarrow \operatorname{Hom}_{B}\left({ }_{B} \mathrm{~S}_{0}(B){ }_{B},{ }_{A} M_{B}\right) \rightarrow \operatorname{Hom}_{B}\left({ }_{B} \mathrm{~S}_{1}(B)_{B},{ }_{A} M_{B}\right) \rightarrow \ldots
$$

Then ${ }_{A} M_{B}$ is isomorphic to $\operatorname{Hom}_{B}\left({ }_{B} \mathrm{~S} \bullet(B)_{B},{ }_{A} M_{B}\right)$ in $\mathrm{D}^{+}\left(\operatorname{Mod} A \otimes_{k} B^{o p}\right)$. By adjointness, for every $n \geq 0$, we have the following isomorphism in Mod $A \otimes_{k} B^{o p}$ :

$$
\operatorname{Hom}_{B}\left({ }_{B} \mathrm{~S}_{n}(B)_{B},{ }_{A} M_{B}\right) \cong \operatorname{Hom}_{k}\left({ }_{B} \mathrm{~S}_{n-1}(B)_{k},{ }_{A} M_{k}\right)
$$

Since $B$ is $k$-projective, by [CE], Chap. IX, Proposition 2.3a, $\operatorname{Hom}_{k}\left({ }_{B} \mathrm{~S}_{n-1}(B)_{k},{ }_{A} I_{k}^{i}\right)$ is injective as a left $A \otimes_{k} B^{o p}$-module for every $n, i \geq 0$. Therefore, for every $n \geq 0$, $\operatorname{Hom}_{k}\left({ }_{B} \mathrm{~S}_{n-1}(B)_{k},{ }_{A} M_{k}\right)$ has the following injective resolution in $\operatorname{Mod} A \otimes_{k} B^{o p}$ :

$$
0 \rightarrow \operatorname{Hom}_{k}\left(B_{B} \mathrm{~S}_{n-1}(B)_{k},{ }_{A} M_{k}\right) \rightarrow \operatorname{Hom}_{k}\left({ }_{B} \mathrm{~S}_{n-1}(B)_{k},{ }_{A} I_{k}^{0}\right) \rightarrow \operatorname{Hom}_{k}\left({ }_{B} \mathrm{~S}_{n-1}(B)_{k},{ }_{A} I_{k}^{1}\right) \rightarrow \ldots
$$

By Lemma 1.1 we complete the proof.

Remark 3.6. In the proof of Theorem 3.5, we cannot induce an $(A-B)$-bimodule morphism $\operatorname{Hom}_{k}\left({ }_{B} \mathrm{~S}_{n-1}(B)_{k},{ }_{A} M_{k}\right) \rightarrow \operatorname{Hom}_{k}\left({ }_{B} \mathrm{~S}_{n}(B)_{k},{ }_{A} M_{k}\right)$ from any morphism of $\operatorname{Hom}_{B}\left({ }_{B} \mathrm{~S}_{n}(B)_{k},{ }_{B} \mathrm{~S}_{n-1}(B)_{k}\right)$ in the case of non-commutative algebras. Then the above complex $V^{\bullet}$ cannot be described by a double complex in general.

For an injective left module $I$ over a ring $R$, we denote by $\operatorname{Ps}(I)$ the category of left $R$-modules which are direct summands of direct products of copies of $I$.

Lemma 3.7. Let $R$ be a ring, $I$ an injective left $R$-module.

(a) If $R$ is a left Noetherian ring, then $\mathrm{Ps}(I)$ is closed under direct sums.

(b) If $R$ is a right coherent ring and $\operatorname{fdim}_{R} I \leq n$, then every $R$-module of $\operatorname{Ps}(I)$ is of flat dimension $\leq n$.

Proof. See [Ma and [CE], Chap. II, Ex. 2.

Proposition 3.8. Let $k$ be a commutative ring, and $R$ a left Noetherian projective $k$-algebra. Then every flat left $R$-module $F$ has the following injective resolution:

$$
0 \rightarrow F \rightarrow I^{0} \rightarrow I^{1} \rightarrow I^{2} \rightarrow \ldots
$$

where $I^{n} \in \operatorname{Ps}\left(\bigoplus_{i=0}^{n} E^{i}\right)$ for all $n \geq 0$.

Proof. By Theorem 3.5] $R$ has the following injective resolution in $R \otimes_{k} R^{o p}-$ Mod:

$$
0 \rightarrow R \rightarrow V^{0} \rightarrow V^{1} \rightarrow V^{2} \rightarrow \ldots
$$

where $V^{n}=\bigoplus_{i=0}^{n} \operatorname{Hom}_{k}\left(\mathrm{~S}_{i-1}(R), E^{n-i}\right)$ for all $n \geq 0$. Since all $\mathrm{S}_{i-1}(R)$ are projective $k$-modules, it is easy to see that every $V^{n}$ belongs to $\operatorname{Ps}\left(\bigoplus_{i=0}^{n} E^{i}\right)$. According to Lemmas 3.3 and 3.7 every flat $R$-module $F$ has the following injective resolution:

$$
0 \rightarrow F \rightarrow I^{0} \rightarrow I^{1} \rightarrow I^{2} \rightarrow \ldots,
$$

where $I^{n} \in \operatorname{Ps}\left(\bigoplus_{i=0}^{n} E^{i}\right)$ for all $n \geq 0$. Hence we get the statement.

Theorem 3.9. Let $k$ be a commutative ring, and $R$ a left Noetherian projective $k$ algebra. Then $\bigoplus_{i=0}^{n} E^{i}$ is an injective cogenerator for the category of left $R$-modules of flat dimension $\leq n$. 
Proof. Let $X$ be a left $R$-module of flat dimension $\leq n$; then $X$ has the following flat resolution :

$$
0 \rightarrow F_{n} \rightarrow \ldots \rightarrow F_{1} \rightarrow F_{0} \rightarrow X \rightarrow 0 .
$$

By Proposition 3.8 every $F_{j}$ has the following injective resolution:

$$
0 \rightarrow F_{j} \rightarrow I_{j}^{0} \rightarrow I_{j}^{1} \rightarrow I_{j}^{2} \rightarrow \ldots,
$$

where $I_{j}^{m} \in \operatorname{Ps}\left(\bigoplus_{i=0}^{m} E^{i}\right)$ for all $m \geq 0$. By Corollary 1.3 , we get an injection from $X$ to $\bigoplus_{i=0}^{n} I_{i}^{i}$. Since $\bigoplus_{i=0}^{n} I_{i}^{i}$ belongs to $\operatorname{Ps}\left(\bigoplus_{i=0}^{n} E^{i}\right)$, we complete the proof.

Corollary 3.10. Let $k$ be a commutative ring, and $R$ a Noetherian projective $k$ algebra which is a finitely generated $k$-module. Then $\bigoplus_{i=0}^{n} E^{i}$ is a $\Sigma$-embedding cogenerator for the category of left $R$-modules of flat dimension $\leq n$. In particular, every indecomposable injective left $R$-module of flat dimension $\leq n$ is a direct summand of $\bigoplus_{i=0}^{n} E^{i}$.

Proof. Since $R$ is a finitely generated $k$-module, we can replace $\operatorname{Ps}\left(\bigoplus_{i=0}^{m} E^{i}\right)$ by $\operatorname{Add}\left(\bigoplus_{i=0}^{m} E^{i}\right)$ in Proposition 3.8. Therefore we can also replace $\operatorname{Ps}\left(\bigoplus_{i=0}^{m} E^{i}\right)$ by $\operatorname{Add}\left(\bigoplus_{i=0}^{m} E^{i}\right)$ in the proof of Theorem 3.9 .

\section{Applications to the Auslander condition}

Let $R$ be a coherent ring. Auslander defined the Auslander condition for $R$ : for every finitely presented left $R$-module $M$ and every $i \geq 0$, every submodule $N$ of $\operatorname{Ext}_{R}^{i}(M, R)$ satisfies that $\operatorname{Ext}_{R}^{j}(N, R)=0$ for all $0 \leq j<i$. This property implies the right side version of it. Moreover, the Auslander condition is equivalent to the following: $\operatorname{fdim}_{R} E^{i} \leq i$ for all $i \geq 0$ (see [FGR] for details). A Noetherian ring $R$ is called Auslander-Gorenstein provided that $R$ satisfies the Auslander condition and that $\operatorname{idim}\left(R_{R}\right)$ and $\operatorname{idm}\left({ }_{R} R\right)$ are finite, and is called Auslander regular if $R$ is an Auslander-Gorenstein ring of which global dimension is finite. For an $R$-module $M$, we denote by $\mathrm{E}(M)$ the injective hull of $M$.

Theorem 4.1. Let $k$ be a commutative ring, and $R$ a right coherent and left Noetherian projective $k$-algebra. Then the following are equivalent.

(a) $R$ satisfies the Auslander condition.

(b) $\operatorname{fdim}_{R} \mathrm{E}(M) \leq \operatorname{fdim}_{R} M$ for every left $R$-module $M$.

Proof. $(a) \Rightarrow(b)$ : We may assume $\operatorname{fdim}_{R} M=n<\infty$. According to Theorem 3.9, $M$ admits an injection to some injective left $R$-module $I$ in $\operatorname{Ps}\left(\bigoplus_{i=0}^{n} E^{i}\right)$. By Lemma 3.7. we have $\operatorname{fdim}_{R} I \leq n$. Since the injective hull $\mathrm{E}(M)$ of $M$ is a direct summand of $I$, we have $\operatorname{fdim}_{R} \mathrm{E}(M) \leq n$.

$(b) \Rightarrow(a)$ : If $\operatorname{fdim}_{R} \operatorname{Im} \delta^{n-1} \leq n$, then $\operatorname{fdim}_{R} E^{n-1} \leq n$ because of $E^{n-1}$ being the injective hull of $\operatorname{Im} \delta^{n-1}$. Moreover, by dimension shift, we have $\operatorname{fdim}_{R} \operatorname{Im} \delta^{n} \leq$ $n+1$. Hence we complete the proof by induction on $n$.

Proposition 4.2. Let $k$ be a commutative ring, and $R$ a Noetherian projective $k$ algebra which satisfies the Auslander condition. Then $\bigoplus_{i=0}^{n} E^{i}$ is a $\Sigma$-embedding cogenerator for the category of left $R$-modules of flat dimension $\leq n$.

Proof. By Theorem 3.9, it is sufficient to show that $\operatorname{Ps}\left(\bigoplus_{i=0}^{n} E^{i}\right)=\operatorname{Add}\left(\bigoplus_{i=0}^{n} E^{i}\right)$. Clearly, $\operatorname{Ps}\left(\bigoplus_{i=0}^{n} E^{i}\right)$ contains $\operatorname{Add}\left(\bigoplus_{i=0}^{n} E^{i}\right)$. Conversely, for every indecomposable injective left $R$-module $I$ of $\operatorname{Ps}\left(\bigoplus_{i=0}^{n} E^{i}\right)$, the Auslander condition implies that 
$\operatorname{fdim}_{R} I$ is at most $n$ by Lemma 3.7. According to [H1], Theorem 6.3, $I$ is a direct summand of $\bigoplus_{i=0}^{n} E^{i}$, and hence $I$ belongs to $\operatorname{Add}\left(\bigoplus_{i=0}^{n} E^{i}\right)$.

Proposition 4.3. Let $k$ be a commutative ring, and $R$ an Auslander-Gorenstein projective $k$-algebra of injective dimension $n$. For a left $R$-module $M$, the following are equivalent.

(a) $\operatorname{fdim}_{R} M \leq m$.

(b) $\operatorname{idim}_{R} M \leq n$ and $M$ has the following minimal injective resolution:

$$
0 \rightarrow M \rightarrow I^{0} \rightarrow I^{1} \rightarrow I^{2} \rightarrow \ldots
$$

where $\operatorname{fdim}_{R} I^{i} \leq \min (i+m, n)$ for all $i \geq 0$.

Proof. $(a) \Rightarrow(b)$ : Let $0 \rightarrow F_{m} \rightarrow \ldots \rightarrow F_{1} \rightarrow F_{0} \rightarrow M \rightarrow 0$ be a flat resolution of $M$. According to [H2], Proposition 6, every flat left $R$-module $F$ is of finite projective dimension. Then, by Corollary 1.3 it is easy to see $\operatorname{idim}_{R} F \leq n$. By Proposition 3.8, it is easy to see that $F_{i}$ has the following injective resolution:

$$
0 \rightarrow F_{i} \rightarrow I_{i}^{0} \rightarrow I_{i}^{1} \rightarrow \ldots \rightarrow I_{i}^{n} \rightarrow 0
$$

where $I^{j} \in \operatorname{Ps}\left(\bigoplus_{i=0}^{j} E^{i}\right)$ for all $j \geq 0$. Then by Corollary 1.3 , $M$ has the following injective resolution:

$$
0 \rightarrow M \rightarrow Q^{0} \rightarrow Q^{1} \rightarrow \ldots \rightarrow Q^{n} \rightarrow 0
$$

where $Q^{0}$ is a direct summand of $\bigoplus_{i=0}^{\min (m, n)} I_{i}^{i}$, and $Q^{j}=\bigoplus_{i=0}^{\min (m, n-j)} I_{i}^{i+j}(j \geq 1)$. Then, for every $0 \leq j \leq n$, we have $Q^{j} \in \operatorname{Ps}\left(\bigoplus_{i=0}^{\min (j+m, n)} E^{i}\right)$. By the definition of Auslander-Gorenstein rings, we have $\operatorname{fdim}_{R} Q^{j} \leq \min (j+m, n)$ for all $j \geq 0$. Since every $j$-th term of a minimal injective resolution of $M$ is a direct summand of $Q^{j}$, the condition $(b)$ is satisfied.

$(b) \Rightarrow(a)$ : Let $0 \rightarrow M \rightarrow I^{0} \rightarrow I^{1} \rightarrow I^{2} \rightarrow \ldots \rightarrow I^{s} \rightarrow 0$ be a minimal injective resolution of $M$, where $s \leq n$. By dimension shifting, we have $\operatorname{fdim}_{R} M \leq m$.

Proposition 4.4. Let $k$ be a commutative ring, and $R$ an Auslander-Gorenstein projective $k$-algebra of injective dimension $\leq n+1$. Then we have $0 \leq \operatorname{fdim}_{R} M-$ $\operatorname{fdim}_{R} \mathrm{E}(M) \leq n$ for every left $R$-module $M$ of finite flat dimension.

Proof. Let $M$ be a left $R$-module of finite flat dimension. By [H2], Proposition 6, we have $\operatorname{fdim}_{R} M \leq n+1$. According to Theorem 4.1 it suffices to consider the case of $\operatorname{fdim}_{R} M=n+1$. Let $M \rightarrow \mathrm{E}(M)$ be the injective hull of $M$; then we have the following short exact sequence:

$$
0 \rightarrow M \rightarrow \mathrm{E}(M) \rightarrow C \rightarrow 0
$$

If $\operatorname{fdim}_{R} \mathrm{E}(M)=0$, then $\operatorname{fdim}_{R} C=n+2$. By [H2], Proposition 6, this is contradiction. Therefore $\operatorname{fdim}_{R} \mathrm{E}(M)>0$, and hence we complete the proof.

Corollary 4.5. Let $k$ be a commutative ring, and $R$ an Auslander regular projective $k$-algebra of injective dimension $\leq n+1$. Then $0 \leq \operatorname{fdim}_{R} M-\operatorname{fdim}_{R} \mathrm{E}(M) \leq n$ for every left $R$-module $M$. 


\section{REFERENCES}

[B1] H. Bass, Injective dimension in Noetherian rings, Trans. Amer. Math. Soc. 102 (1962), 18-29. MR 25:2087

[B2] H. Bass, On the ubiquity of Gorenstein rings, Math. Z. 82 (1963), 8-28. MR 27:3669

[BN] M. Bökstedt and A. Neeman, Homotopy limits in triangulated categories, Compositio Math. 86 (1993), 209-234. MR 94f: 18008

[CE] H. Cartan and S. Eilenberg, "Homological algebra," Princeton Univ. Press, Princeton, NJ, 1956. MR 17:1040e

[FGR] R. M. Fossum, P. A. Griffith and I. Reiten, "Trivial extensions of abelian categories," Lecture Notes in Math., Vol. 456, Springer-Verlag, Berlin, 1975. MR 52:10810

[H1] M. Hoshino, Noetherian rings of self-injective dimension two, Comm. Algebra 21 (1993), 1071-1094. MR 94a:16010

[H2] M. Hoshino, On self-injective dimensions of Artinian rings, Tsukuba J. Math. 18 (1994), 1-8. MR 95g:16006

[HS] B. Huisgen-Zimmermann and S. O. Smalø, A homological bridge between finite and infinite dimensional representations of algebras, preprint.

[L] D. Lazard, Autour de la platitude, Bull. Soc. Math. France 97 (1968), 81-128. MR 40:7310

[Ma] E. Matlis, Injective modules over Noetherian rings, Pacific J. Math. 8 (1958), 511-528. MR 20:5800

[Mi] J. Miyachi, Duality for derived categories and cotilting bimodules, J. Algebra 185 (1996), 583-603. MR 98b:18016

[X] J. Xu, Minimal injective and flat resolutions of modules over Gorenstein rings, J. Algebra 175 (1995), 451-477. MR 96h:13025

Department of Mathematics, Tokyo Gakugei University, Koganei-Shi, Tokyo 1848501, JAPAN

E-mail address: miyachi@u-gakugei.ac.jp 